\title{
Accumulation of a soluble form of human nectin-2 is required for exerting the resistance against herpes simplex virus type 2 infection in transfected cells
}

\author{
Y. FUIMOTO ${ }^{1,2^{*}}$, K. OZAKI ${ }^{2}$, N. IWAMORI ${ }^{1,2}$, H. TAKAKUWA ${ }^{3}$, E. ONO ${ }^{1,2^{*}}$
}

\begin{abstract}
${ }^{1}$ Department of Biomedicine, Graduate School of Medical Sciences, Kyushu University, Fukuoka 812-8582, Japan; ${ }^{2}$ Center of Biomedical Research, Research Center for Human Disease Modeling, Graduate School of Medical Sciences, Kyushu University, Fukuoka 812-8582, Japan; ${ }^{3}$ Faculty of Life Sciences, Kyoto Sangyo University, Kyoto 603-8555, Japan
\end{abstract}

\begin{abstract}
Summary. - Cell entry of herpes simplex virus type 2 (HSV-2) requires the interaction of viral glycoprotein D (gD) with the receptor nectin- 1 and herpesvirus entry mediator (HVEM). In addition, it is known that nectin-2 is also functional as a receptor for HSV-2, although the binding to the gD is weak. To examine an antiviral potential of a soluble form of human nectin-2 (hNectin-2Ig), transfected Vero cells expressing the entire ectodomain of nectin-2 fused to the Fc portion of human IgG were established. Specific binding of hNectin-2Ig to HSV-2 gD was confirmed by ELISA. Competitive ELISA demonstrated that accumulation of hNectin-2Ig in transfected cells increased significantly in a cell culture time dependent manner. Viral growth of several HSV-2 strains was significantly inhibited in the transfected cells that were cultured for $72 \mathrm{hr}$ compared with control Vero cells, but not in cells that were cultured for $24 \mathrm{hr}$. These results indicate that accumulation of a soluble form of nectin-2 is required for exerting the resistance against HSV-2 infection.
\end{abstract}

Keywords: antiviral effect; soluble form of nectin-2; herpes simplex virus type 2

\section{Introduction}

Herpes simplex virus (HSV), a representative member of the subfamily Alphaherpesvirinae, commonly infects the skin, eyes and the mucous membrane in regions of the mouth and genitalia (Spear et al., 2000). HSV type 2 (HSV-2) causes genital diseases and neonatal infections through a maternal genital tract to the newborn (Pellet and Roizman, 2001). Genital herpes caused by HSV-2 is one of the most important sexually transmitted diseases (Bystrická et al., 2003).

*Corresponding authors. E-mail: yosikazu@anim.med.kyushuu.ac.jp (Y. Fujimoto), etsuro@anim.med.kyushu-u.ac.jp (E. Ono), phone: +81-92-642-6159.

Abbreviations: $\mathrm{HSV}=$ herpes simplex virus; $\mathrm{gD}=$ glycoprotein $\mathrm{D}$; HVEM = herpes virus entry mediator; hNectin- $2 \mathrm{~h}=$ human nectin-2; Nectin-2Ig = soluble form of human nectin-2; gD2$\mathrm{Ig}=$ soluble form of HSV-2 gD; p.i. = post infection
It is known that several envelope glycoproteins including $\mathrm{gB}, \mathrm{gC}, \mathrm{gD}, \mathrm{gH}$, and $\mathrm{gL}$ require for efficient HSV entry into target cell interaction with host cell surface molecules. The initial interaction of gB and gC to cell surface 3-O-sulfated heparin sulfate (3-O-S-SH) promotes an efficient viral entry into the cells (Herold et al., 1994). Subsequently, the binding of $\mathrm{gB}$ and $\mathrm{gD}$ to receptor on cell surface can mediate fusion of viral envelope with the cellular membrane (Spear et al., 2000). Receptors for gD like herpesvirus entry mediator (HVEM), 3-O-S-SH, nectin-1, and nectin-2 have been well characterized (Spear et al., 2000). Nectin-1 and HVEM are competent receptors for both HSV-1 and HSV-2 entry, while nectin-2 is a receptor for HSV-2 only (Warner et al., 1998; Lopez et al., 2000; Martinez et al., 2001). Nectin-1 and nectin-2 are expressed individually or together in various human cells of neuronal, fibroblastic, and epithelial origin (Warner et al., 1998; Guzman et al., 2006). In these cell types, each or both of these proteins could be important entry mediators for HSV-2 strains. Although nectin-2 has been examined from a functional perspective as a receptor for HSV-2 gD, 
there has been no report showing a detailed mechanism of nectin-2 mediated viral entry into cells.

We previously reported that resistance to HSV-1 infection was conferred by expression of a soluble form of HVEM or porcine nectin-1 in transfected cells and transgenic mouse cell lines (Ono et al., 2004a,b,c). In the present study, we have established a stable cell line expressing a soluble form of human nectin-2 (hNectin-2) and examined the antiviral potential against HSV-2 infection.

\section{Materials and Methods}

Viruses and cells. HSV-1 VR3 (ATCC VR-539) strain, which was kindly provided by Dr. T. Suzutani, and HSV-2 KN, YS-1, YS-4 (Sakuma et al., 1988) and 186 (Rawls et al., 1968) strains, which were kindly provided by Drs. Y. Yanagi and H. Minagawa, were propagated in Vero cells. All of HSV-2 strains were clinical isolates. Strain $\mathrm{KN}$ was isolated from genital herpetic lesion of an adult female and, strains YS-1 and YS-4 were isolated from vesicular skin lesions on the face of an infant. Strains KN and YS-1, but not YS-4, were sensitive to acyclovir (Sakuma et al., 1988). Strain 186 was isolated from a genital lesion from an individual attending a sexually transmitted disease clinic in Houston, Texas, in the 1960s (Rawls et al., 1968). The strain 186 had severe pathogenic impact on mice as compared to strains KN, YS-1 and YS-4. Vero and CHO-K1 cells were grown in Dulbecco's modified Eagle's medium (DMEM, Gibco) containing $5 \%$ fetal bovine serum (FBS) at $37^{\circ} \mathrm{C}$ in $5 \% \mathrm{CO}_{2}$.

Construction of plasmids and stable transfection. To construct a plasmid expressing hNectin-2, the cDNA of full-length open reading frame was amplified by PCR from pENTR221 plasmid containing a complete hNectin-2 gene (pENTR221/nectin-2; GenBank Acc. No.: DQ891829, GeneCopoeia). Amplified cDNA fragment was cloned into pCXN2 expression vector (Niwa et al., 1991) containing neomycin resistance gene. CHO-K1 cells were transfected with the plasmid using METAFECTENE PRO (Biontex Laboratories), and then cultured in DMEM containing $800 \mu \mathrm{g} / \mathrm{ml}$ of G418 (Roche) to establish a stable cell line expressing human nectin-2 (hNectin-2/CHO). To construct plasmids expressing soluble forms of human nectin-2 (hNectin-2Ig) and HSV-2 gD (gD2-Ig) we used gene of extracellular domain (hNectin-2: aa 1-350 and HSV-2 gD: aa 1-339) and of Fc portion of human IgG1. Extracellular domains amplified by PCR were cloned into a plasmids carrying IgG1-Fc DNA (Nakagawa et al., 1998), and then chimeric gene fragment was inserted into $\mathrm{pCXN} 2$ vector as described previously (Ono et al., 2004a). pENTR221/nectin-2 and HSV-2 genome DNA were used as templates for PCR. Genome DNA of HSV-2 strain 186 was extracted from infected Vero cells using QIAamp DNA mini kit (Qiagen). PCR primer sets used for construction of plasmids are as follows: 5'-GGACCCGT CGACCCACCATGGCCCGGGCCGC-3' and 5'-CTGCGCGT CGACAATCTACACATAAACTGCCCGCCG-3' for hNectin-2, 5'-GGACCCCTCGAGCCACCATGGCCCGGGCCGC-3' and
5'-CTGAGCGGGATCCTCTCGGACAAAGATGACC-3' for hNectin-2Ig, and 5'-CCGCTCGAGCACCATGGGGCGTTTGA CCTC-3' and 5'-CGAAGATCTGGGTTGCTGGGGGCGGCG-3' for gD2-Ig. Vero cells were transfected with each constructed plasmid as described above. To confirm the secretion of each fusion protein, the cultured supernatants from transfectants were collected and protein G-Sepharose $4 \mathrm{~B}$ (Sigma) added and incubated for $3 \mathrm{hr}$ at $4^{\circ} \mathrm{C}$. The beads were washed with phosphate-buffered saline (PBS) and used for Western blot analysis with alkaline phosphatase-conjugated goat anti-human IgG Fc specific antibody (1:3,000, Sigma) for the detection of hNectin-2Ig or gD2-Ig, and mouse anti-HSV-1/2 gD antibody (1:3,000, Santa Cruz) and alkaline phosphatase-conjugated goat anti-mouse IgG antibody (1:3,000, Sigma) for the detection of gD2-Ig.

Flow cytometry. To confirm expression of hNectin-2 on the cell surface of hNectin-2/CHO cell line, the cells were stained with monoclonal mouse anti-nectin-2 (1:100, Sigma) and FITCconjugated goat anti-mouse IgG antibodies (1:100, Sigma), and analyzed by using a FACSCalibur (Becton Dickinson). Data were analyzed by CellQuest Pro software (Becton Dickinson).

Immunofluorescence analysis. Expression of soluble form of nectin-2 in the transfectants was confirmed by double immunofluorescence staining using monoclonal mouse anti-nectin-2 (1:100, Sigma) and FITC-conjugated goat anti-mouse IgG antibodies (1:100, Sigma) for nectin-2, and TRITC-conjugated goat antihuman IgG (Fc specific) antibodies (1:100, Sigma) for Fc region of human IgG. Indirect immunofluorescence assay was performed to confirm HSV infection in hNectin-2/CHO cells. Cells were infected with viruses at $\mathrm{MOI}$ of 10 , fixed after $24 \mathrm{hr}$ and stained with mouse anti-HSV-1/2 gD (1:100, Santa Cruz) and FITC-conjugated goat anti-mouse IgG specific antibodies.

Virus infection in transformed cells expressing the hNectin-2Ig. For the investigation of viral growth kinetics, the Vero cells were seeded in $35 \mathrm{~mm}$-diameter dishes $\left(2 \times 10^{5}\right.$ cells/dish). The cells were infected by HSV-1 and HSV-2 at MOI of 0.001 at $37^{\circ} \mathrm{C}$ for 1 $\mathrm{hr}$, washed and cultured in $4 \mathrm{ml}$ of DMEM. Five hundred microliters of supernatant were harvested from the infected cells at $24 \mathrm{hr}$ intervals, and titration of viral infectivity was performed on Vero cells. The infective dose was calculated by the method of Reed and Muench (1938). Plaque assay of HSV-2 infections with $30 \mathrm{PFU}$ on $35 \mathrm{~mm}$-diameter dishes were performed as described previously (Ono et al., 2004b). The plaque numbers in the hNectin-2Ig/Vero were presented as the relative value to those of control Vero cells, which were defined as $100 \%$. The mean values of the relative dose and standard deviation in plaque assay were determined by triplicate experiments. Statistical analysis was performed by Student's $t$-test.

Binding assay. Soluble forms of hNectin-2 and HSV-2 gD were harvested from culture supernatants of hNectin2-Ig/Vero and gD2-Ig/Vero cell lines, and purified by the affinity chromatography with protein G-Sepharose 4B. To examine the binding of hNectin2Ig to gD2-Ig, an ELISA was performed. Briefly, $50 \mu \mathrm{l}$ of purified hNectin-2Ig $(5 \mu \mathrm{g} / \mathrm{ml})$ was immobilized onto 96 -well plate. After 
blocking with $10 \%$ goat serum in tris-buffered saline (TBS), 30 $\mu \mathrm{l}$ of different concentrations of purified gD2-Ig were added to the wells and incubated at room temperature for $1 \mathrm{hr}$. The wells were washed with TBS containing $0.05 \%$ Tween 20 and primary mouse anti-HSV-1/2 gD antibodies $(1: 5,000)$ were added for $1 \mathrm{hr}$. After washing, the secondary alkaline phosphatase conjugated anti-mouse IgG specific antibodies (1:5,000) were added and after $1 \mathrm{hr}$, the wells were washed and Alkaline phosphatase substrate kit (Bio-Rad) was used to detect binding of gD2-Ig to hNectin-2Ig according to the manufacturer's instructions. The absorbance at $405 \mathrm{~nm}$ was measured using SpectraMax 190 microplate reader (Molecular Devices).

Competitive enzyme-linked immunosorbent assay. Cells were seeded into $60 \mathrm{~mm}$-diameter dishes $\left(2 \times 10^{6}\right.$ cells/dish $)$ and cultured in DMEM containing 5\% FBS for 8, 24, 48 and $72 \mathrm{hr}$. The cells were washed with PBS and lysed with $1 \mathrm{ml}$ of RIPA buffer (ATTO). To measure concentration of hNectin-2Ig in the transfected cells, competitive ELISA using purified hNectin-2-Ig was performed as described previously (Ono et al., 2006). Concentrations of total proteins were determined by Bradford protein assay (Bio-Rad) according to the manufacturer's instructions. At indicated time point, the number of cells grown in parallel dish was counted by trypan blue staining.

Neutralization test. The neutralizing activity of purified hNectin2Ig against HSV-2 infections was determined. Each serial 3-folddiluted hNectin-2Ig of which starting concentration was $1 \mathrm{mg} / \mathrm{ml}$ was mixed with $100 \mathrm{PFU}$ of HSV-2 and incubated at $4^{\circ} \mathrm{C}$ for $2 \mathrm{hr}$. Subsequently, the mixture was inoculated on Vero cells seeded into 96 -well plate and kept at $37^{\circ} \mathrm{C}$ for $1 \mathrm{hr}$. The cells were washed and cultured for 2 days to observe the cytopathic effect by virus infections.

\section{Results}

Earlier, it was reported that hNectin-2 served as an entry receptor for HSV-2 strains, but not for wild type of HSV-1 strains (Struyf et al., 2002). To confirm the recep-

\section{(a)}
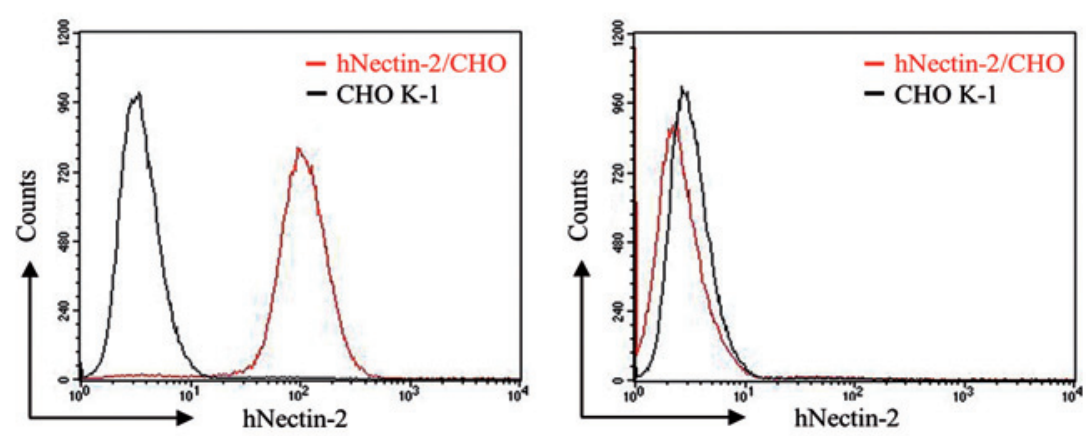

(b)
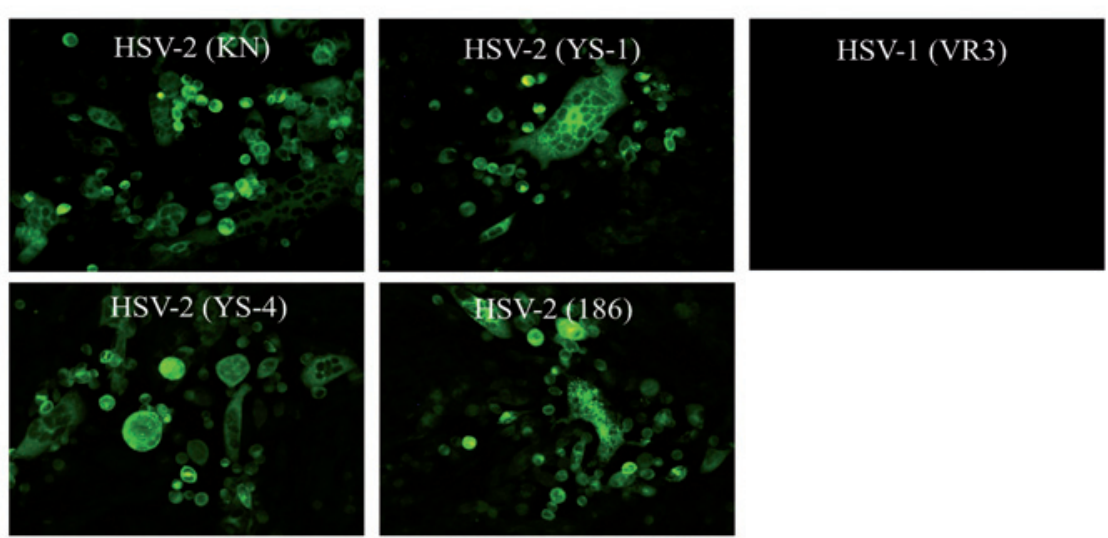

Fig. 1

Establishment of transfected cells expressing hNectin-2

(a) Flow cytometry of hNectin-2/CHO and CHO K-1 cells stained with monoclonal anti-nectin-2 (left panel), and cells stained by only FITC-conjugated goat anti-mouse IgG antibody as a control (right panel). (b) hNectin-2/CHO cells were inoculated with HSV-2 strains KN, YS-1, YS-4, and 186 and HSV-1 strain VR3. At $24 \mathrm{hr}$ post infection (p.i.), the cells were stained with anti-HSV-1/2 gD antibodies and FITC-conjugated goat anti-mouse IgG antibodies. 100x magnified. 
tor activity of hNectin-2 expressed from the constructed plasmid, CHO-K1 cells lacking HSV gD receptors (Krummenacher et al., 2004) were transfected. Transfected CHO cells were inoculated with several strains of HSV-2 and HSV-1. Expression of hNectin-2 on the cell surface of the transfected $\mathrm{CHO}$ cells were confirmed by flow cytometry analysis (Fig. 1a). The indirect immunofluorescence assay using anti-HSV-1/2 gD antibody revealed that all of tested HSV-2 strains KN, YS-1, YS-4, and 186 infected cells expressing nectin-2, but the cells could not be infected by HSV-1 strain VR3 (Fig.1b). These results demonstrate that HSV-2 interacts with nectin-2 expressed on cell surface and enters into the transfected CHO cells.

We previously showed that transfected Vero cells expressing a soluble form of porcine nectin-1 exhibited significant resistance to HSV-1 infection (Ono et al., 2004b). As described above, nectin-2 is utilized by HSV-2 as an entry receptor. To examine whether a soluble form of nectin- 2 confers the antiviral potential to Vero cells, five transfected cell lines expressing a fusion protein of extracellular domain of hNectin-2 and the Fc portion of human IgG1 (hNectin-2Ig) were established (Fig. 2a). In all of the established cell lines, strong fluorescent signals were detected in their cytoplasm by immunofluorescence analysis using anti-human IgG antibodies (data not shown). To further confirm the expression of hNectin-2Ig, Western blot analysis and double-immunofluorescence staining were performed. In Western blot analysis of supernatant of one of the established cell lines, an anti-nectin-2 specific band was detected at a molecular weight of about 70 $\mathrm{K}$ corresponding to the size of hNectin-2Ig (Fig. 2b). In this cell line, fluorescent signals for nectin-2 and human IgG co-localized in the cytoplasm (Fig. 2c). This cell line was designated as hNectin-2Ig/Vero, and used for further studies.

To confirm the interaction of hNectin-2Ig with HSV-2 gD, a binding assay was performed using purified soluble form of HSV-2 gD (gD2-Ig: a fusion protein of the extracellular

(a)

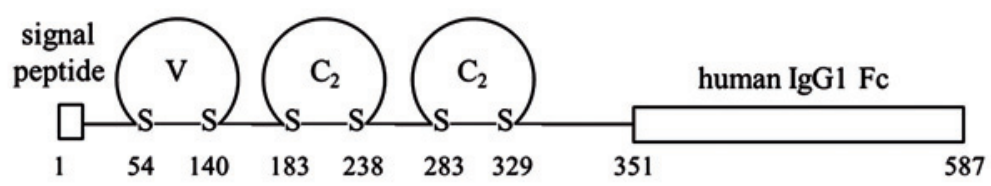

(b)

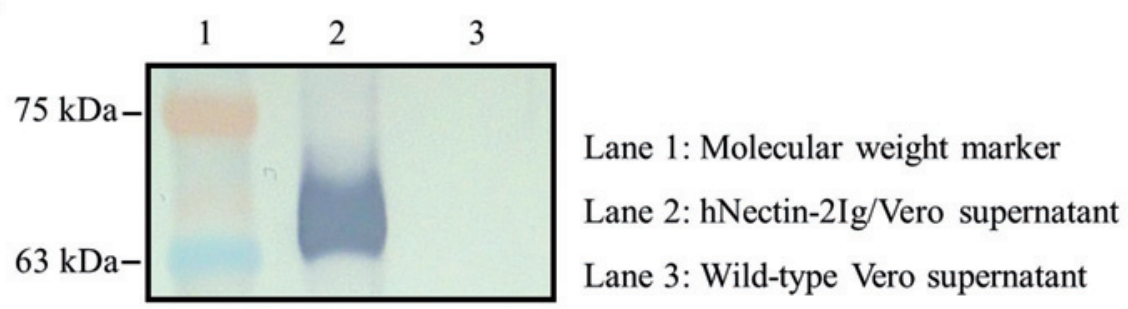

(c)

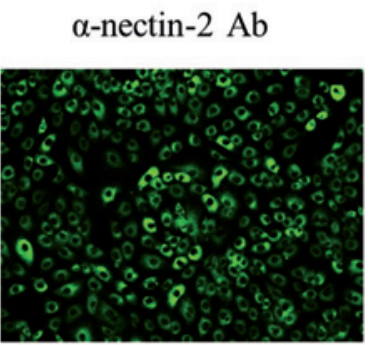

$\alpha$-human IgG Ab

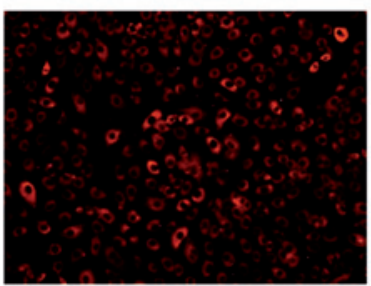

Fig. 2

Establishment of transfected cells expressing hNectin-2Ig

(a) Schematic representation of hNectin-2Ig consisting of the entire ectodomain of hNectin-2 (aa 1-350) and the Fc portion of human IgG (aa 351-587). (b) Detection of the fusion protein secreted in culture supernatant of hNectin-2Ig/Vero and control Vero cells by Western blot analysis using anti-human IgG Fc specific antibody. (c) Double immunofluorescence staining of hNectin-2Ig/Vero using anti-nectin-2 ( $\alpha$-nectin-2 Ab) and FITC-conjugated goat anti-mouse IgG antibodies (green), and TRITC-conjugated goat anti-human IgG Fc specific antibody (red). 100x magnified. 
(a)

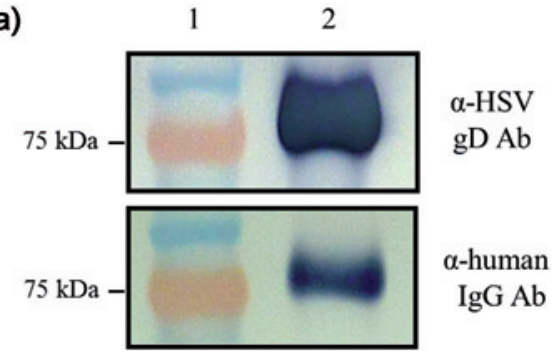

Lane 1: Molecular weight marker

Lane 2: Purified gD2-Ig

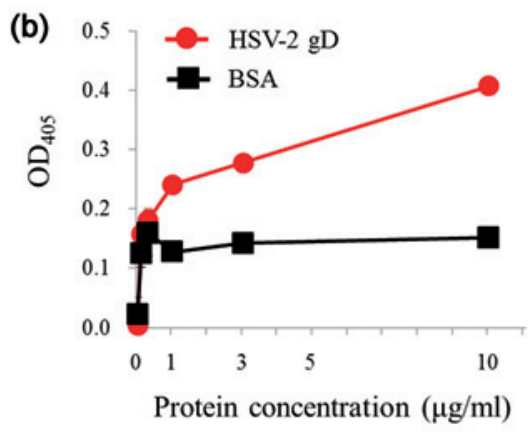

Fig. 3

Binding of hNectin-2Ig to HSV-2 gD

(a) Detection of purified soluble form of HSV-2 gD by Western blot analysis using anti-HSV-1/2 gD (upper figure) and anti-human IgG Fc antibodies (lower figure). (b) Affinity-purified hNectin-2Ig was immobilized on 96-well plates, and then incubated with different amounts of purified gD2-Ig (red circles) and BSA as a control protein (black squares). The averages and standard deviations of absorbance values in triplicate experiments were done.

domain of HSV-2 gD and human IgG Fc) and hNectin-2Ig that was purified from the supernatant of hNectin-2Ig/ Vero cell line. In Western blot analysis, a band of gD2-Ig was detected by both of anti-HSV-1/2 gD and anti-human IgG antibodies (Fig. 3a). As can be seen in Fig. 3b, the binding of (a)
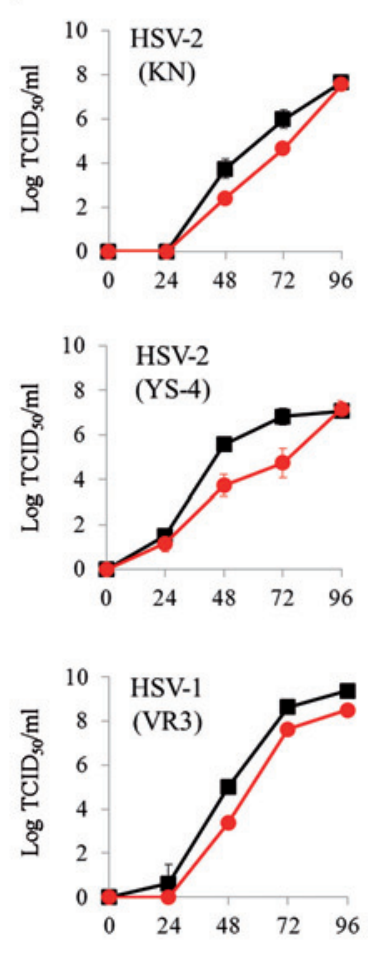

Hours post infection
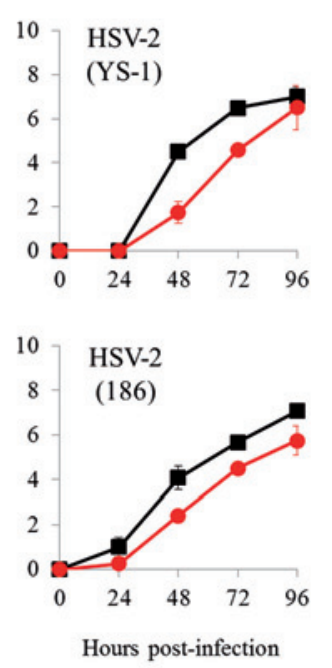

hNectin-2Ig/Vero

Vero (b)
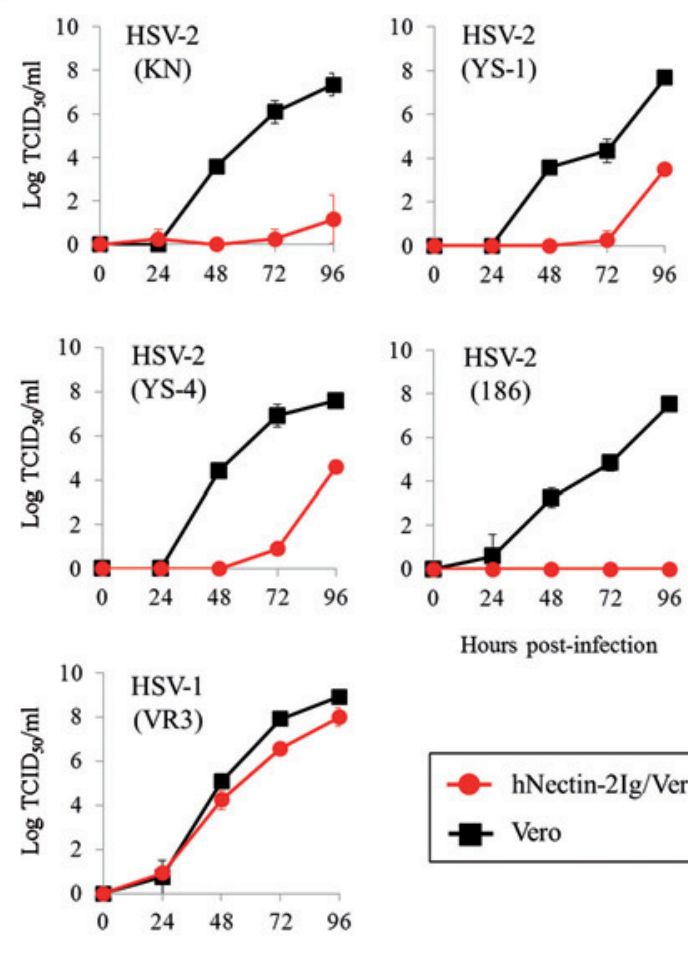

hNectin-2Ig/Vero

Vero

Fig. 4

Multi-cycle growth curves of HSV-2 and HSV-1 strains in transfected cells expressing hNectin-2Ig hNectin-2Ig/Vero and control Vero cells at 24 (a) or $72 \mathrm{hr}$ (b) after seeding of the cells infected with HSV-2 strains KN, YS-1, YS-4, and 186, and HSV-1 strain. Each culture supernatant of hNectin-2Ig/Vero (red circles) and control Vero cells (black squares) were collected at the indicated time points. Viral titers were determined on Vero cells and the mean viral titers of independent triplicates are shown. 
hNectin-2Ig to gD2-Ig was detectable in a dose-dependent fashion, but not observed with the control protein.

It has already been known that soluble forms of nectin-1 block HSV-1 infection (Geraghty et al., 1998; Krummenacher et al., 1998; Ono et al., 2004a,b). We have performed the neutralization test against HSV-2 infections using purified hNectin-2Ig. Since concentration of hNectin-2Ig excreted from hNectin-2Ig/Vero after $72 \mathrm{hr}$ culture was approximately $1 \mathrm{mg} / \mathrm{ml}$ (data not shown), we prepared $1.0 \mathrm{mg} / \mathrm{ml}$ of purified hNectin-2Ig solution for the neutralization test. Even the highest concentration of hNectin-2Ig $(1.0 \mathrm{mg} / \mathrm{ml})$ did not inhibit the infection with neither of used HSV-2 strains, indicating that the direct binding of hNectin-2Ig to HSV-2 $\mathrm{gD}$ is not sufficient to neutralize HSV-2 infection because of the low affinity of hNectin-2Ig to gD.

To assess the antiviral potential of hNectin-2Ig to HSV-2 infection, we investigated the growth kinetics of several HSV-2 strains in hNectin-2Ig/Vero and control Vero cells. Twenty-four hours after seeding of the cells, both cell lines were infected with HSV-2 (strains KN, YS-1, YS-4, or 186) at MOI of 0.001 , and viral titers in cultured supernatants 24, 48, 72, $96 \mathrm{hr}$ p.i. were determined. Growth curves of all HSV-2 strains used in hNectin-2Ig/Vero cell line were similar to those in control Vero cells, although viral titers in hNectin-2Ig/Vero cell line 48 and 72 hr p.i. were slightly lower than control Vero cells (Fig. 4a). As a control experiment, hNectin-2Ig/Vero cell line was infected with HSV-1 strain VR3, because it has been known that nectin-2 does not serve as an entry receptor for wild type HSV-1 (Struyf et al., 2002). As expected, the growth curve of HSV-1 in hNectin-2Ig/Vero cell line was similar to that in control Vero cells (Fig. 4a). In the immunofluorescence analysis, it was found that the intensities of fluorescent signals were variable in cytoplasm of hNectin-2Ig/Vero cells $24 \mathrm{hr}$ after seeding of the cells (Fig. 5b), suggesting that there is variability in expression level of hNectin-2Ig among the transfected cells that were cultured for $24 \mathrm{hr}$. Furthermore, it was also found that number of the cells possessing stronger fluorescent signal was increased in a manner dependent on the culture time (Fig. 5a/d). Additionally, competitive ELISA also demonstrated that the amount of hNectin-2Ig in the transfected cells increased significantly depending on the culture time (Fig. 5e). To determine the antiviral potential of accumulating hNectin-2Ig, hNectin-2Ig/Vero and control Vero cells were cultured for $72 \mathrm{hr}$ and inoculated with HSV-2 and HSV-1 strains. As shown in Fig. 4b, viral growth of all HSV-2 strains used in this study was inhibited in hNectin-2Ig/Vero cell line, but not in control Vero cells. On the other hand, hNectin-2Ig/Vero cell line did not show the antiviral effect against the HSV-1 strain. Furthermore, in the plaque assay $72 \mathrm{hr}$ after seeding of the cells, the plaque formation of all HSV-2 strains was inhibited in hNectin-2Ig/Vero cell line as compared to control Vero cells, but not after $24 \mathrm{hr}$ (Fig. 6). Taken together, these results demonstrated that accumulation of a soluble form of nectin-2 is required for exerting the antiviral effect to HSV-2 infection.
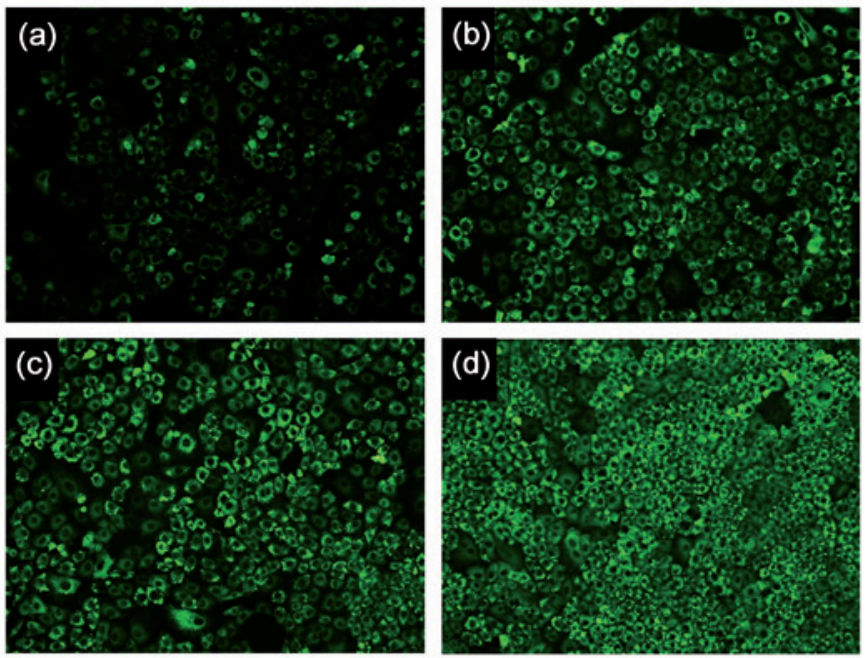

Fig. 5 (e)

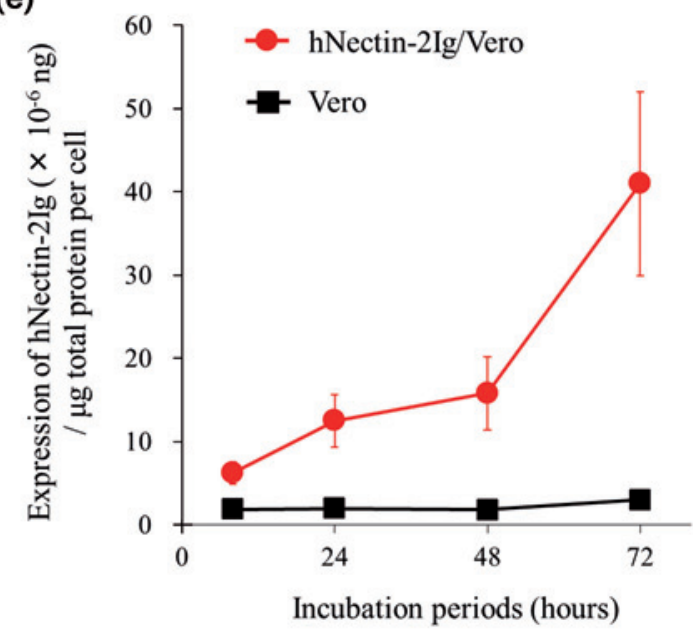

Accumulation of hNectin-2Ig in transfected cells

Immunofluorescence staining of hNectin-2Ig/Vero using FITC-conjugated goat anti-human IgG antibody 8 (a), 24 (b), 48 (c) and 72 hr (d) after seeding the cells. (e) Competitive ELISA of accumulation of hNectin-2Ig in the transfected cells. The transfected cells were lysed at the indicated time points, and each concentration of hNectin-2Ig was measured. The averages and standard deviations of hNectin-2Ig concentration per $1 \mu \mathrm{g}$ of total proteins per cell were determined by triplicate experiments. 


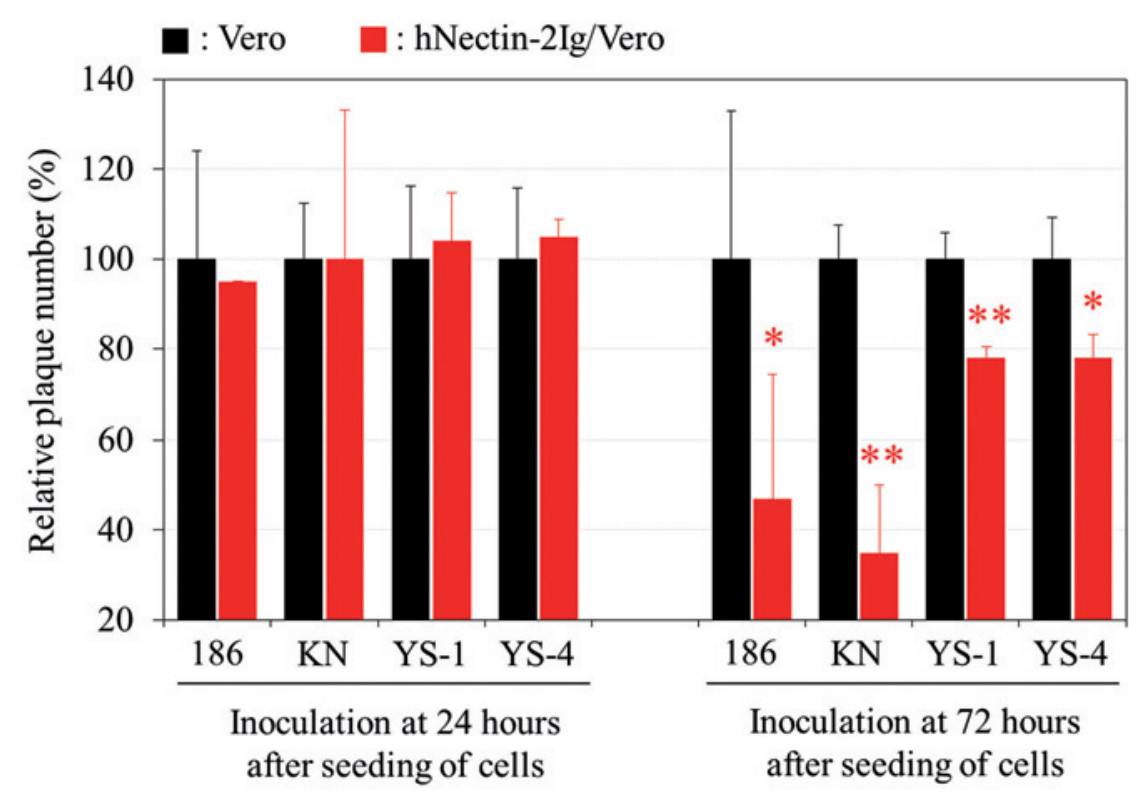

Fig. 6

Plaque assay of HSV-2 infection in transfected cells expressing hNectin-2Ig

hNectin-2Ig/Vero and control Vero cells 24 or $72 \mathrm{hr}$ after seeding were infected with 30 PFU of HSV-2 strains KN, YS-1, YS-4, and 186. The mean values of the relative plaque number were determined by triplicate experiments. ${ }^{*}$ and ${ }^{\star *}$ indicate $\mathrm{P}<0.05$ and $\mathrm{P}<0.01$, respectively.

\section{Discussion}

Previous studies demonstrated that nectin-2 enabled the entry of HSV-2 into target cells (Martinez and Spear, 2001; Struyf et al., 2002). Consistent with these studies, CHO cell line expressing hNectin-2 was susceptible to HSV-2 infection in the present study. To examine the antiviral potential of a soluble form of hNectin-2 against HSV-2 infections, we established transfected Vero cells expressing hNectin-2Ig that was confirmed to bind to HSV-2 gD. However, purified hNectin-2Ig with a high concentration did not neutralize HSV-2 infection, indicating that the direct binding of hNectin-2Ig to HSV-2 gD is not sufficient to neutralize HSV-2 infection. We assume that in the neutralization assay, after pre-incubation with hNectin-2Ig, the virus was inoculated into Vero cells that expressed the intact nectin-1 on the cell surface and gD may have released hNectin-2Ig and bind to nectin-1 because of its affinity differences, resulting in HSV-2 infection.

Experimental infections showed that the inhibition of HSV-2 growth was observed in the transfected cells that were cultured for $72 \mathrm{hr}$ to accumulate hNectin-2Ig in the cells, but not in the transfected cells that were cultured for $24 \mathrm{hr}$. There are two possible effects of hNectin-2Ig on the suppression of HSV-2 replication. Firstly, Cocchi et al. (2000) reported that nectin-2 $\alpha$ (a soluble form of nectin-2) promotes cell-to-cell spread of an HSV-1 gD mutant. Excessive accumulation of hNectin-2Ig into intracellular space may block the cell-to-cell spread of HSV-2, in addition to HSV-1 gD mutants. Secondly,
gD newly-synthesized in the rough endoplasmic reticulum of infected cells with HSV-2 is transported to the transmembrane via secretary pathway including the Golgi apparatus (Johnson and Spear, 1983; Murata et al., 2002). Since hNectin-2Ig was secreted into medium, the molecules passed through the secretary pathway of the transfected cells. Therefore, hNectin-2Ig might have many opportunities to bind to newly synthesized gD during the transport, resulting in viral assembly and blocking the infection of transfected cells. In our previous reports, it has been considered that the antiviral effects of soluble forms of HVEM and porcine nectin-1 were mainly due to their direct binding to gD of HSV-1 (Ono et al., 2004a,b,c). Therefore, antiviral mechanism of hNectin-2Ig against HSV-2 infections was apparently different from those of soluble forms of HVEM and nectin-1. The present study suggested that there were several mechanisms in the antiviral effects of soluble forms of $\mathrm{gD}$ receptors against $\mathrm{HSV}$ infections.

Acknowledgement. We thank Dr. J. Miyazaki for providing pCXN2, Dr. T. Suzutani for HSV-1 VR3 strain, and Dr. Y. Yanagi and Dr. H. Minagawa for HSV-2 strains.

\section{References}

Bystrická M, Kriková Z, Kŕcová M, Koncová K, Kovácová E, Vaculíková A, Staneková D, Russ G (2003): Sexually transmitted infections among prostitutes in Bratislava, Slovakia. Acta Virol. 47, 167-172. 
Cocchi F, Menotti L, Dubreuil P, Lopez M, Campadelli-Fiume G (2000): Cell-to-cell spread of wild-type herpes simplex virus type 1 , but not of syncytial strains, is mediated by the immunoglobulin-like receptors that mediate virion entry, nectin1 (PRR1/HveC/HIgR) and nectin2 (PRR2/ HveB). J. Virol. 74, 3909-3917. http://dx.doi.org/10.1128/ JVI.74.8.3909-3917.2000

Geraghty RJ, Krummenacher C, Cohen GH, Eisenberg RJ, Spear PG (1998): Entry of alphaherpesviruses mediated by poliovirus receptor-related protein 1 and poliovirus receptor. Science 280, 1618-1620. http://dx.doi.org/10.1126/ science. 280.5369 .1618

Guzman G, Oh S, Shukla D, Engelhard HH, Valyi-Nagy T (2006): Expression of entry receptor nectin-1 of herpes simplex virus 1 and/or herpes simplex virus 2 in normal and neoplastic human nervous system tissues. Acta Virol. 50, 59-66.

Herold BC, Visalli RJ, Susmarski N, Brandt CR, Spear PG (1994): Glycoprotein C-independent binding of herpes simplex virus to cells requires cell surface heparan sulphate and glycoprotein B. J. Gen. Virol. 75, 1211-1222. http://dx.doi. org/10.1099/0022-1317-75-6-1211

Johnson DC, Spear PG (1983): O-linked oligosaccharides are acquired by herpes simplex virus glycoproteins in the Golgi apparatus. Cell 32, 987-997. http://dx.doi. org/10.1016/0092-8674(83)90083-1

Krummenacher C, Baribaud F, Ponce de Leon M, Baribaud I, Whitbeck JC, Xu R, Cohen GH, Eisenberg RJ (2004): Comparative usage of herpesvirus entry mediator A and nectin- 1 by laboratory strains and clinical isolates of herpes simplex virus. Virology 322, 286-299. http:// dx.doi.org/10.1016/j.virol.2004.02.005

Krummenacher C, Nicola AV, Whitbeck JC, Lou H, Hou W, Lambris JD, Geraghty RJ, Spear PG, Cohen GH, Eisenberg RJ (1998): Herpes simplex virus glycoprotein D can bind to poliovirus receptor-related protein 1 or herpesvirus entry mediator, two structurally unrelated mediators of virus entry. J. Virol. 72, 7064-7074.

Lopez M, Cocchi F, Menotti L, Avitabile E, Dubreuil P, CampadelliFiume G (2000): Nectin2alpha (PRR2alpha or HveB) and nectin2delta are low-efficiency mediators for entry of herpes simplex virus mutants carrying the Leu25Pro substitution in glycoprotein D. J. Virol. 74, 1267-1274. http://dx.doi.org/10.1128/JVI.74.3.1267-1274.2000

Martinez WM, Spear PG (2001): Structural features of nectin-2 (HveB) required for herpes simplex virus entry. J. Virol. 75, 11185-11195. http://dx.doi.org/10.1128/ JVI.75.22.11185-11195.2001

Murata T, Goshima F, Takakuwa H, Nishiyama Y (2002): Excretion of herpes simplex virus type 2 glycoprotein $\mathrm{D}$ into the culture medium. J. Gen. Virol. 83, 2791-2795. http:// dx.doi.org/10.1099/0022-1317-83-11-2791

Nakagawa I, Murakami M, Ijima K, Chikuma S, Saito I, Kanegae Y, Ishikura H, Yoshiki T, Okamoto H, Kitabatake A, Uede $\mathrm{T}$ (1998): Persistent and secondary adenovirus-mediated hepatic gene expression using adenovirus vector containing CTLA4IgG. Hum. Gene Ther. 9, 1739-1745. http:// dx.doi.org/10.1089/hum.1998.9.12-1739
Niwa H, Yamamura K, Miyazaki J (1991): Efficient selection for high-expression transfectants with a novel eukaryotic vector. Gene 108, 193-199. http://dx.doi.org/10.1016/03781119(91)90434-D

Ono E, Amagai K, Taharaguchi S, Tomioka Y, Yoshino S, Watanabe Y, Cherel P, Houdebine LM, Adam M, Eloit M, Inobe M, Uede T (2004a): Transgenic mice expressing a soluble form of porcine nectin-1/herpesvirus entry mediator $\mathrm{C}$ as a model for pseudorabies-resistant livestock. Proc. Natl. Acad. Sci. USA 101, 16150-16155. http://dx.doi.org/10.1073/pnas.0405816101

Ono E, Amagai K, Yoshino S, Taharaguchi S, Inobe M, Uede T (2004b): Resistance to pseudorabies virus infection in transformed cell lines expressing a soluble form of porcine herpesvirus entry mediator C. J. Gen. Virol. 85, 173-178. http://dx.doi.org/10.1099/vir.0.19481-0

Ono E, Yoshino S, Amagai K, Taharaguchi S, Kimura C, Morimoto J, Inobe M, Uenishi T, Uede T (2004c): Enhanced resistance to herpes simplex virus type 1 infection in transgenic mice expressing a soluble form of herpesvirus entry mediator. Virology 320, 267-275. http://dx.doi.org/10.1016/j. virol.2003.11.031

Ono E, Tomioka Y, Watanabe Y, Amagai K, Taharaguchi S, Glenisson J, Cherel P (2006): The first immunoglobulin-like domain of porcine nectin-1 is sufficient to confer resistance to pseudorabies virus infection in transgenic mice. Arch. Virol. 151, 1827-1839. http://dx.doi.org/10.1007/ s00705-006-0747-6

Pellet PE, Roizman B (2001): The Family Herpesviridae: A brief introduction. In Knipe DM, Howley P, Griffin DE, Lamb RA, Martin MA, Roizman B, Straus SE (Eds): Fields Virology. 5th ed., Lippincott-Williams and Wilkins, New York, N.Y. pp. 2479-2499.

Rawls WE, Laurel D, Melnick JL, Glicksman JM, Kaufman RH (1968): A search for viruses in smegma, premalignant and early malignant cervical tissues. The isolation of Herpesviruses with distinct antigenic properties. Am. J. Epidemiol. 87, 647-655.

Reed LJ, Muench H (1938): A simple method of estimating fifty per cent endpoints. Am. J. Epidemiol. 27, 493-497.

Sakuma S, Yamamoto M, Kumano Y, Mori R (1988): An acyclovirresistant strain of herpes simplex virus type 2 which is highly virulent for mice. Arch. Virol. 101, 169-182. http:// dx.doi.org/10.1007/BF01310998

Spear PG, Eisenberg RJ, Cohen GH (2000): Three classes of cell surface receptors for alphaherpesvirus entry. Virology 275, 1-8. http://dx.doi.org/10.1006/viro.2000.0529

Struyf F, Martinez WM, Spear PG (2002): Mutations in the N-terminal domains of nectin- 1 and nectin-2 reveal differences in requirements for entry of various alphaherpesviruses and for nectin-nectin interactions. J. Virol. 76, 12940-12950. http://dx.doi.org/10.1128/JVI.76.24.12940-12950.2002

Warner MS, Geraghty RJ, Martinez WM, Montgomery RI, Whitbeck JC, Xu R, Eisenberg RJ, Cohen GH, Spear PG (1998): A cell surface protein with herpesvirus entry activity (HveB) confers susceptibility to infection by mutants of herpes simplex virus type 1 , herpes simplex virus type 2 , and pseudorabies virus. Virology 246, 179-189. http:// dx.doi.org/10.1006/viro.1998.9218 